\title{
Avaliação da apraxia em nonagenários: dados de um ambulatório de geriatria
}

\author{
Apraxia evaluation in nonagenarian: data from a geriatric outpatient clinic
}

\author{
Juliana Francisca Cecatoa, José Eduardo Martinellib \\ a Psicóloga. Especialista em Psicopedagogia pela Faculdade de Medicina de Jundiaí (FAJ). Mestre em Ciências da Saúde (FMJ). Professora colaboradora da disciplina de Geriatria e \\ Gerontologia da FMJ, departamento de clínica médica.

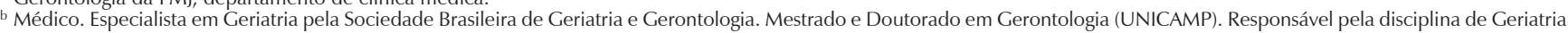 \\ e Gerontologia da FMJ, departamento de clínica médica.
}

RESUMO

ABSTRACT
Introdução: Apraxia é comumente descrita como um sintoma da doença de Alzheimer (DA). Seu aparecimento em pacientes nonagenários é pouco conhecida.

Objetivo: Avaliar e comparar o desempenho de nonagenários portadores de DA e idosos não portadores em subtestes para apraxia.

Materiais e Métodos: Foram avaliados 78 indivíduos, com graus variados de escolaridade e com 90 anos ou mais de idade, por meio de Miniexame do Estado Mental (MEEM), Cambridge Cognitive Examination (CAMCOG) e o Questionário de Atividades Funcionais de Pfeffer (QAFP). Para analisar a presença de apraxia, foram selecionados oito subitens do CAMCOG: os desenhos do Pentágono, da espiral, da casa, do relógio, e também a tarefa de colocar um pedaço de papel em um envelope; avaliação dos movimentos corretos com uma mão para dar "adeus", cortar papel com uma tesoura e escovar os dentes. O diagnóstico da DA foi estabelecido de acordo com o National Institute of Neurological and Communicative Disorders and Stroke and The Alzheimer's disease and Related Disorders Association (NINCDS-ADRDA).

Resultados: Encontrou-se para o MEEM média de 18,48 pontos, para o CAMCOG 59,94 pontos e nos subitens Apraxia 8,65 pontos no grupo que recebeu diagnóstico de DA, enquanto que os sem DA (grupo controle) apresentou média no MEEM de 26,15 pontos, CAMCOG 82,23 pontos e Apraxia de 9,96 pontos. Estas diferenças são estatisticamente significativas entre os grupos nos testes, respectivamente, $p<0,0001, p<0,0001$ e $p=0,007$. Um coeficiente de correlação moderado negativo e significativo pode ser encontrado entre Apraxia e QAFP $(r=-0,51 ; p=0,0003)$.

Conclusão: A presença de apraxia em nonagenários mostrou valores significativamente maiores naqueles portadores de DA em relação aos não portadores.

Palavras-chave: apraxias; testes neuropsicológicos; idoso; doença de Alzheimer; nonagenários.

ABSTRACT

Introduction: It is common to describe Apraxia as a symptom of Alzheimer's disease (AD). Its appearance in nonagenarian patients is little known.

Objective: To evaluate and compare the performance of nonagenarian patients with $A D$ and healthy elderly in the subtests for apraxia. Materials and Methods: We evaluated 78 people with varying degrees of education and 90 years of age or older, through the Mini-Mental State Examination (MMSE), Cambridge Cognitive Examination (CAMCOG) and Pfeffer Functional Activities Questionnaire (PFAQ). To analyze the presence of apraxia, eight sub-itens of CAMCOG were selected: the drawings of the pentagon, spiral, house, clock; the tasks of putting a piece of paper in an envelope; evaluation of the correct movements with one hand to give "goodbye", cutting paper with scissors and brush teeth. AD diagnostic was established according to National Institute of Neurological and Communicative Disorders and Stroke and The Alzheimer's disease and Related Disorders Association (NINCDS-ADRDA)

Results: We observed an average MMSE of 18.48 points, CAMLOG 59.94 points and 8.65 points in the sub-itens for Apraxia in the group diagnosed with AD, while the control group had a mean MMSE of 26.15 points, CAMLOG 82 and Apraxia of 9.96 points. Therefore, there are statistically significant differences between the groups in the tests, respectively, $p<0.0001, p<0.0001$ e $p=0.007$. A negative moderate and significant correlation coefficient was found between apraxia and PFAQ ( $r=-0.51 ; p=0.0003)$.

Conclusion: The presence of in nonagenarian showed significantly higher values in patients with AD compared to controls.

Keywords: apraxias; neuropsychological tests; aged; Alzheimer's disease; nonagenarians.

\section{Correspondência: \\ JULIANA F. CECATO \\ Rua Prudente de Moraes, 111 - VI Argos \\ cep 00000-000 Jundiaí, SP, Brasil \\ E-mail: cecatojuliana@hotmail.com} Exceto onde especificado diferentemente, a matéria publicada neste periódico é licenciada sob forma de uma licença Creative Commons BY-NC 4.0 Internacional. http://creativecommons.org/licenses/by-nc/4.0/ 


\section{INTRODUÇÃO}

Em 2011 a população idosa brasileira era de 20,5 milhões, o equivalente a $10,8 \%$ da população total ${ }^{1}$. Vale ainda destacar o expressivo aumento da população com idade superior a 80 anos. No Brasil, o conjunto dos idosos tem aumentado em torno de $3,5 \%$ ao ano, enquanto que o número de idosos com mais de 80 anos tem crescido $4,7 \%$ ao ano $^{2}$. Esta alteração demográfica tem um impacto significativo sobre as condições de saúde da população, com repercussões importantes para o sistema de saúde pública ${ }^{3}$.

Atualmente, os especialistas no estudo do envelhecimento referem-se a três grupos de pessoas mais velhas: os idosos jovens, os idosos velhos e os idosos mais velhos. O termo "idosos jovens" refere-se às pessoas de 65 a 74 anos. O grupo "idosos velhos" está na faixa etária de 75 a 84 anos e os "idosos mais velhos" são aqueles de 85 anos ou mais e tem maior tendência para incapacidades e enfermidades e podem ter dificuldades para desempenhar as atividades de vida diária (AVDs)

O aumento significativo na faixa da população com 85 anos ou mais veio mostrar que não dispomos de dados normativos de alta qualidade em domínios cognitivos entre várias de suas áreas: memória, apraxia (habilidades motoras) e percepção (agnosia). Enquanto os transtornos de memória e linguagem tendem a dominar a neurologia cognitiva e a neuropsicologia, os distúrbios práxicos tem ficado num segundo plano, acarretando muitas vezes dificuldades na correta interpretação da verdadeira natureza dos distúrbios motores apresentados por pacientes portadores de doenças neurológicas e neuropsiquiátricas as mais variadas 5 .

A apraxia é a incapacidade de realizar tarefas motoras voluntárias aprendidas ao longo da vida, ou seja, incapacidade de realizar um ato complexo de maneira correta, sendo progressiva na doença de Alzheimer (DA) como sinal de comprometimento do lobo parietal dominante. A apraxia não se deve a fraqueza muscular, dificuldade de compreensão ou falta de coordenação, e sim as lesões em áreas cerebrais responsáveis pelo planejamento e sequenciamento das funções motoras. Todas as funções práxicas dependem de áreas parietais de associação onde os centros psicomotores planejam a função motora dos atos aprendidos ${ }^{6,7}$.

Um dos modelos da neuropsicologia cognitiva bastante difundido para a construção de instrumentos que se propõem avaliar as habilidades práxicas é o sugerido por Rothi et al. ${ }^{8}$. De acordo com esse modelo, os movimentos são mediados por distintos subsistemas, e a execução, imitação e compreensão dos movimentos podem ser deficitárias independentemente. Os autores consideram que há dois léxicos relacionados à ação: entrada (memória dos movimentos reconhecidos) e saída realização dos movimentos. Essa divisão foi idealizada a partir da identificação de pacientes com dificuldade para imitar gestos, mas não para produzi-los após ordem verbal ${ }^{9}$.

Neste modelo postula-se ainda a presença de uma via não léxica responsável pela execução de gestos não familiares, sem registro prévio pela via lexical. Por sua vez, os gestos representacionais, como o se despedir por um aceno, fazem parte do léxico de saída incorporados a um sistema semântico, em que há o reconhecimento da ação e como ela deve ser executada ${ }^{10}$.

A formulação de uma ação requer a vontade e a intenção de realizar tal tarefa, e isto é gerado no córtex pré-frontal. Esse sinal é então utilizado para ativar os sistemas frontoparietais no hemisfério dominante que por sua vez ativam os engramas motores (por exemplo, o padrão e sequência dos movimentos necessários para acender um fósforo) no córtex pré-motor. Estes engramas motores são encaminhados para o córtex motor primário e daí através dos tratos corticoespinais até o músculo com modulações do cerebelo e dos núcleos da base ${ }^{11}$.

A apraxia é uma função cognitiva que se relaciona à capacidade de realizar e/ou imitar movimentos aprendidos (não instintivos) e habilidosos. Em outras palavras, constitui a "memória do ato motor". Sua disfunção, denomina-se dispraxia, refere-se a uma dificuldade de realizar ações motoras, a qual não pode ser explicada isoladamente pelo não entendimento da tarefa (afasia de compreensão), pela não colaboração (negativismo), dificuldade de força muscular (paresia ou plegia) ou perda da propriocepção ${ }^{11,12}$.

A idade é o principal fator de risco para o desenvolvimento da DA, portanto a prevalência desta entidade aumenta com o envelhecimento. A DA é a causa mais frequente de demência, estando presente em $60 \%$ dos casos, tanto antes como depois dos 65 anos, idade em que se estabeleceu o limite arbitrário entre demência de início precoce e demência de início tardio ${ }^{13}$.

A apresentação clínica inicial mais comum da DA é o déficit de memória episódica, acompanhada do comprometimento progressivo de outros domínios cognitivos como afasia, apraxia ou incoordenação visuomotora espacial $^{10,11}$. Historicamente, Alois Alzheimer em 1907 e Pick em 1906 já haviam mencionado as apraxias como uma das funções cognitivas afetadas nos pacientes com demência.

Atualmente as apraxias se incluem dentro dos critérios diagnósticos de demência do DSM-IV como sendo um dos domínios cognitivos possível de se alterar na enfermidade, condição que se mantem no DSM-V $\mathrm{V}^{14}$. A apraxia é progressiva na DA como sinal de comprometimento do lobo parietal dominante. A apraxia é consequência de lesões em áreas cerebrais responsáveis pelo planejamento 
e sequenciamento das funções motoras. Todas as funções práxicas dependem das áreas parietais de associação onde os centros psicomotores planejam a função motora dos atos aprendidos ${ }^{6}$.

A apraxia é comumente descrita como um sintoma da DA e é um dos sinais clínicos que distingue esta e outras demências corticais daquelas que envolvem primariamente estruturas subcorticais, embora tenha sido relatada em doenças neurodegenerativas subcorticais e lesões vasculares ${ }^{15}$. Três das principais formas de apraxia são ideomotora, ideacional e a construcional. Na apraxia ideomotora a pessoa é incapaz de realizar tarefas aprendidas quando recebe os objetos necessários para ela. Por exemplo, ela pode usar uma chave inglesa como uma caneta, enquanto que na apraxia ideacional a pessoa não consegue fazer tarefas complexas na ordem correta, uma dificuldade poderia ser colocar a meia depois do sapato por exemplo. A apraxia construcional é a incapacidade de construir figuras geométricas, montar quebra-cabeças ou desenhar um cubo ou outras figuras geométricas (por exemplo, ele é incapaz de fazer um desenho com molde) $)^{16-18}$.

O Cambridge Mental Disorders of the Elderly Examination (CAMDEX) é um instrumento de avaliação diagnóstica desenvolvido como uma entrevista estruturada com o propósito de diagnosticar e quantificar a demência em idosos ${ }^{19}$. Utilizamos o CAMDEX como instrumento diagnóstico na análise dos nossos pacientes e através da sua bateria cognitiva (Cambridge Cognitive Examination CAMCOG) avaliamos dentre os vários aspectos da cognição a apraxia construcional, ideomotora e ideacional ${ }^{20}$. Assim, utilizamos os subtestes da bateria cognitiva do CAMDEX que avaliam apraxia para comparar o desempenho de nonagenários portadores de DA e idosos não portadores em subtestes para apraxia.

\section{MATERIAIS E MÉTODOS}

Esta pesquisa foi realizada no Ambulatório de Geriatria e Gerontologia da Faculdade de Medicina de Jundiaí, no período de janeiro de 2011 a Dezembro de 2014. Trata-se de um estudo de corte transversal, que contou com 78 participantes, de ambos os sexos, com graus variados de escolaridade e com 90 anos ou mais de idade. Esta pesquisa foi aprovada pelo Comitê de Ética da Faculdade de Medicina de Jundiaí, com o parecer 54/11.

Todos os pacientes passam por anamnese clínica detalhada e exame neurológico geral que permita conhecer a situação neurológica real do paciente assim como a sua mão dominante. Em seguida, são submetidos a vários testes neuropsicométricos: Cambridge Cognitive Examination $\left(\right.$ CAMCOG $^{19}$ e o Miniexame do Estado Mental (MEEM) ${ }^{20}$.
Também foram aplicadas a Escala de Depressão Geriátrica $(\text { EDG })^{21}$ e o Questionário de Atividades Funcionais de Pfeffer $(\mathrm{QAFP})^{22}$.

O CAMCOG (bateria cognitiva do CAMDEX) avalia as funções cognitivas através de 67 itens (memória, atenção, concentração, orientação, linguagem, pensamento abstrato, cálculo, praxia e percepção) com uma pontuação máxima de 107 pontos, sendo o ponto de corte para demência 80 pontos $^{19}$.

O Miniexame do Estado Mental (MEEM) ${ }^{20}$ tem sua pontuação máxima de 30 pontos, e seu ponto de corte varia de acordo com a escolaridade: analfabetos 20 pontos, 1 a 4 anos de estudo 25 pontos, 5 a 8 anos 26 pontos, 9 a 11 anos 28 pontos e 29 pontos para $>11$ anos de estudo ${ }^{23}$.

O diagnóstico de demência foi corroborado pelos critérios do National Institute of Neurological and Communicative Disorders and Stroke and The Alzheimer's disease and Related Disorders Association ${ }^{23}$. Adotamos como critérios de exclusão pacientes com diagnóstico de outras demências, Parkinsonismo primário e secundário, Acidente Vascular Encefálico, Síndrome depressiva e demências reversíveis, com menos de 90 anos, analfabetos e aqueles que não aceitaram participar da pesquisa.

A EDG serviu para diferenciarmos os pacientes portadores de depressão que influenciaria nos resultados assim como o QAFP foi um teste adicional para o diagnóstico de demência.

Para a análise da apraxia, foram selecionados oito subitens do CAMCOG: os desenhos do pentágono (1 ponto), da espiral (1 ponto), da casa (1 ponto), do relógio (3 pontos), e também a tarefa de colocar um papel dentro de um envelope ( 3 pontos) e os movimentos corretos com uma das mãos de dar "adeus", cortar com uma tesoura e escovar os dentes (3 pontos), como demonstra a Tabela 1. A pontuação total dos subitens que avaliam apraxia é igual a 12 (dentre os 107 pontos do total do CAMCOG). Pontuação inferior a 12 é considerada dispraxia.

Para descrever o perfil da amostra segundo as variáveis em estudo foram feitas tabelas de frequência com valores de frequência absoluta (n) e relativa (\%) das variáveis categóricas (sexo e escolaridade), e calculadas as estatísticas descritivas (com medidas de posição e dispersão - média, desvio-padrão, valores mínimo e máximo) das variáveis numéricas (idade e escores dos testes). Para comparar as variáveis categóricas entre os 2 grupos foram usados o teste qui-quadrado $\left(\chi^{2}\right)$ e o teste exato de Fisher (para valores esperados menores que 5). Para comparação das variáveis numéricas entre os 2 grupos foi utilizado o teste de Mann-Whitney, devido à ausência de distribuição Normal da variável. Os testes de normalidade usados foram Shapiro-Wilk e KolmogorovSmirnov. Para analisar a relação entre o escore de praxia e as 
Tabela 1. Subitens do CAMCOG que envolvem a avaliação da praxia.

\begin{tabular}{|c|c|c|}
\hline Subitem & Pontuação & Tipo da apraxia \\
\hline Desenho do pentágono & 1 & \multirow{4}{*}{ Visuoconstrutivas } \\
\hline Desenho da espiral & 1 & \\
\hline Desenho de uma casa em três $D$ & 1 & \\
\hline Desenho de um relógio & 3 & \\
\hline Colocar um papel dentro de um envelope & 3 & Ideacional \\
\hline Fazer o movimento com a mão de dar "tchau" & 1 & \multirow{3}{*}{ Ideomotora } \\
\hline Fazer o movimento com a mão de cortar com uma tesoura & 1 & \\
\hline Fazer o movimento com a mão de escovar os dentes & 1 & \\
\hline Total & 12 & \\
\hline
\end{tabular}

variáveis numéricas foi utilizado o coeficiente de correlação de Spearman, devido à ausência de distribuição Normal da variável. O nível de significância adotado para os testes estatísticos foi de $5 \%(p<0.05)$.

\section{RESULTADOS}

A amostra foi composta por 78 idosos, com média de idade igual a 91,97 anos (mínimo=90; máximo=103; desvio padrão $[d p]=2,44)$, dos quais $62,8 \%(n=49)$ eram do sexo feminino. Em relação aos anos de estudo, $64,1 \%(n=50)$ tinham entre 1 a 4 anos de estudo, $17,9 \%(n=14)$ entre 5 a 8 anos e $17,9 \%(n=14)$ dos participantes tinham 9 ou mais anos. Receberam diagnóstico de DA 66,7\% $(n=52)$ e fizeram parte do grupo controle (GC) 33,3\% ( $n=26)$. De acordo com o teste estatístico Kolmogorov-Smirnov (KS) e Shapiro-Wilk verificaram que a maioria das variáveis não apresentaram distribuição normal e, portanto, foram usados testes nãoparamétricos nas análises.

A Tabela 2 descreve a média de idade no grupo com DA, ligeiramente inferior quando comparado ao grupo controle (GC), com valores iguais a, respectivamente, 91,8 anos e 92,35 anos $(p=0,773)$. Participantes com diagnóstico de demência apresentaram $71,2 \%$ de mulheres, enquanto que no grupo controle verificaram-se maiores frequências de homens $(53,8 \%)(p=0,032)$. A amostra possui um maior número de idosos com baixa escolaridade, com $69,2 \%$ dos participantes com escolaridade entre 1 a 4 anos de estudo $(p=0,830)$.

Optou-se por comparar a média dos escores dos testes cognitivos (MEEM, CAMCOG e Apraxia) entre os grupos DA e GC. Observa-se na Tabela 3, média no MEEM de 18,48 pontos, CAMCOG 59,94 pontos e Apraxia 8,65 pontos no grupo que recebeu diagnóstico de DA, enquanto que o GC apresentou, respectivamente, 26,15 pontos $(p<0,0001)$, 82,23 pontos $(p<0,0001)$ e 9,96 pontos $(p=0,007)$.
Tabela 2. Características entre os grupos diagnósticos com relação a idade, gênero e escolaridade.

\begin{tabular}{lccc}
\hline & DA & GC & $\boldsymbol{p}$ \\
Idade (anos) & $91,80( \pm 2,00)$ & $92,35( \pm 3,15)$ & $* 0,773$ \\
Gênero & & & \\
$\quad$ Feminino & $71,2 \%$ & $46,2 \%$ & $* * \mathbf{0 , 0 3 2}$ \\
$\quad$ Masculino & $28,8 \%$ & $53,8 \%$ & \\
Escolaridade & & & \\
Grupo 1: 1 a 4 anos & $61,5 \%$ & $69,2 \%$ & \\
Grupo 2: 5 a 8 anos & $19,2 \%$ & $15,4 \%$ & $* * * 0.830$ \\
Grupo 3: $>9$ anos & $19,2 \%$ & $15,4 \%$ & \\
\hline
\end{tabular}

* $p=$ Mann-Whitney; $* * p=\chi^{2} ; * * * p=$ exato de Fisher.

Tabela 3. Dados descritivos dos testes MEEM, CAMCOG e Apraxia dos 52 pacientes com diagnóstico de Doença de Alzheimer e dos 26 idosos que fizeram parte do Grupo Controle.

\begin{tabular}{|c|c|c|c|}
\hline & 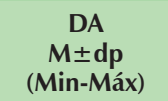 & 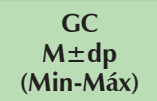 & $p$ \\
\hline MEEM & $\begin{array}{c}18,48 \pm 4,87 \\
\quad(9-28)\end{array}$ & $\begin{array}{c}26,15 \pm 2,15 \\
(22-29)\end{array}$ & 0,0001 \\
\hline CAMCOG & $\begin{array}{c}59,94 \pm 15,49 \\
(23-88)\end{array}$ & $\begin{array}{c}82,23 \pm 7,44 \\
(68-99)\end{array}$ & 0,0001 \\
\hline Apraxia & $\begin{array}{c}8,65 \pm 1,99 \\
(4-12)\end{array}$ & $\begin{array}{c}9,96 \pm 1,48 \\
(7-12)\end{array}$ & 0,007 \\
\hline
\end{tabular}

$p=$ Mann-Whitney; $M$ =média; Min=mínimo; Máx=Máximo; $d p=$ desvio padrão.

Analisando o coeficiente de correlação de Spearman em participantes com escolaridade entre 1 a 4 anos, os resultados apontam para um coeficiente de correlação moderado positivo e significativo entre a apraxia e o MEEM $(r=0,55 ; p<0,0001)$ e CAMCOG $(r=0,67 ; p<0,0001)$. Um coeficiente de correlação moderado negativo e significativo pode ser encontrado entre Apraxia e QAFP $(r=-0,51$; $p=0,0003)$. De acordo com a escolaridade entre 5 a 8 anos, verificam-se coeficientes de correlação moderado positivo e significativo entre a apraxia e o MEEM $(r=0,65 ; p=0,008)$ e 
Tabela 4. Análises do coeficiente de correlação entre apraxia e as variáveis idade, testes cognitivos e atividade funcional, separados por níveis de escolaridade.

\begin{tabular}{|c|c|c|c|c|c|}
\hline & & Idade & MEEM & CAMCOG & QAFP \\
\hline \multicolumn{6}{|c|}{ Escolaridade entre 1 a 4 anos } \\
\hline \multirow{3}{*}{ Apraxia } & $r$ & $-0,18$ & 0,55 & 0,67 & $-0,51 *$ \\
\hline & $p$ & 0,197 & 0,0001 & 0,0001 & 0,0003 \\
\hline & N & 50 & 50 & 50 & 50 \\
\hline \multicolumn{6}{|c|}{ Escolaridade entre 5 a 8 anos } \\
\hline \multirow{3}{*}{ Apraxia } & $r$ & $-0,11$ & 0,65 & 0,66 & $-0,34$ \\
\hline & $p$ & 0,702 & 0,008 & 0,010 & 0,257 \\
\hline & $\mathrm{n}$ & 14 & 14 & 14 & 14 \\
\hline \multicolumn{6}{|c|}{ Escolaridade $>9$ anos } \\
\hline \multirow{3}{*}{ Apraxia } & $r$ & $-0,002$ & 0,004 & 0,28 & $-0,31$ \\
\hline & $p$ & 0,993 & 0,987 & 0,339 & 0,379 \\
\hline & $n$ & 14 & 14 & 14 & 14 \\
\hline
\end{tabular}

$r=$ Spearman; $\mathrm{n}=$ número de sujeitos.

CAMCOG $(r=0,66 ; p=0,010)$. As análises do coeficiente de correlação com participantes com mais de 9 anos de estudo não evidenciaram correlações significativas (Tabela 4).

\section{DISCUSSÃO}

Os subitens de apraxia tiveram pontuação média maior nos idosos saudáveis com significância estatística. Isto se justifica, devido as alterações cerebrais estruturais que ocorrem no paciente com DA (demência cortical com predomínio têmporo-parietal), onde o comprometimento do córtex parietal é o responsável pelas alterações dispráxicas. A apraxia não se correlacionou com a idade por termos uma amostra homogênea entre os grupos. A apraxia e o QAFP se correlacionaram de modo negativo moderado e significativo implicando que quando os idosos apresentam dispraxias, independentemente do seu tipo, surgem dificuldades nas atividades de vida diária contribuindo para a perda de independência.

Estudos sobre a progressão e prognóstico na DA tem mostrado que os pacientes que desenvolvem precocemente apraxia declinam mais rapidamente que aqueles que não apresentam apraxia ${ }^{25,26}$. Esse fato deve-se a inúmeros fatores, sendo um dos mais importantes a degeneração dos lobos têmporo-parietais de forma mais intensa em alguns pacientes do que em outros. Sabemos que a evolução da doença depende de cada pessoa. Em uns evolui de forma mais lenta, enquanto que em outros é extremamente rápida, de tal forma que a DA faz parte do diagnóstico diferencial das demências rapidamente progressivas. Os pacientes não portadores de DA também apresentaram alterações dispráxicas. Estas podem ser explicadas pelas alterações decorrentes do processo de envelhecimento normal sem comprometimento cognitivo. Isso se deve ao fato de apresentarem uma disfunção da região pré frontal caracterizada por discreto prejuízo das funções cognitivas envolvendo um comprometimento leve, porém significativo da memória operacional, apresentando uma maior dificuldade de codificar novas informações.

A apraxia construtiva exige habilidades visuais e planejamento motor. Ambos os hemisférios cerebrais atuam na realização das tarefas construtivas. As falhas estão mais relacionadas a lesões parietais do hemisfério direito por causa do déficit perceptual, enquanto os erros na execução são relacionados a lesões no hemisfério esquerdo. A apraxia ideomotora é relacionada a lesões no córtex parietal do hemisfério esquerdo, no corpo caloso e nos núcleos da base. A apraxia ideacional é geralmente causada por graves perturbações na sequência temporal dos atos motores ${ }^{5}$.

Em nosso trabalho, três tipos de apraxia foram avaliados: a visuoconstrutiva através do desenho do pentágono, da espiral, da casa em 3D e do relógio; a ideacional através de colocar uma folha de papel dentro de um envelope; e a ideomotora através do movimento com a mão de dar tchau, com a mão de cortar com uma tesoura e de escovar os dentes. Apenas $17,9 \%$ da nossa amostra tinham escolaridade maior que 9 anos. O que representa uma das limitações do estudo já que os itens de apraxia visuoconstrutiva sofrem influência da escolaridade, destacando-se o teste do desenho do relógio. As habilidades manuais encontradas em alguns nonagenários, principalmente nos de menor escolaridade, para realizar atividades construtivas, como a escrita ou cópia de modelos são outro fator limitante do estudo (ou um viés de pesquisa). Isto porque muitos deles ficam meses sem escrever ou copiar uma figura e quando o fazem, tem grandes dificuldades comparativamente àqueles que escrevem com frequência. Devemos considerar o grau de escolaridade: quanto menor o nível maior a dificuldade. Este 
fato está de acordo com os achados do nosso estudo onde a escolaridade entre 1 a 4 anos apontou um coeficiente de correlação moderada positiva e significativa entre Apraxia, MEEM e CAMCOG (Tabela 4).

A avaliação da apraxia em nonagenários é uma ferramenta para explorar a área da cognição no diagnóstico das doenças neurodegenerativas que mais acometem essa faixa etária. Utilizamos os itens que avaliam apraxia no CAMCOG e constatamos que elas estão prejudicadas nos pacientes com DA, mas também verificamos comprometimento nos sem DA, talvez em função do envelhecimento normal que afeta área pré-frontais. Novas pesquisas estão em andamento no sentido de avaliar apenas apraxia fora do contexto do CAMCOG ou de qualquer outra entrevista estruturada utilizando ferramentas já elaboradas para essa finalidade.

\section{REFERÊNCIAS}

1. Kuchemann BA. Envelhecimento populacional, cuidado e cidadania: velhos dilemas e novos desafios. Soc Estado. 2012;27(1):165-80. http://dx.doi.org/10.1590/S0102-69922012000100010

2. Batista AS, Jaccoud LB, Aquino L, El-Moor PD. Envelhecimento e dependência: desafios para a organização da proteção social. Brasília: MPS; 2008.

3. Lima-Costa MF, Matos DL, Camargos VP, Macinko J. Tendências em dez anos das condições de saúde de idosos brasileiros: evidências da Pesquisa Nacional por Amostra de Domicílios. Ciênc Saúde Coletiva. 2011;16(9):3689-96. http://dx.doi.org/10.1590/S141381232011001000006

4. Papalia DE, Olds SW, Feldman RD. Desenvolvimento humano. Porto Alegre: Artmed; 2006.

5. Malloy-Diniz L, Fuentes D, Mattos P, Abreu N. Avaliação neuropsicológica.Porto Alegre: Artmed; 2010.

6. Guimarães RM, Cunha UGV. Sinais e sintomas em geriatria. $2 \underline{a}$ ed. São Paulo: Atheneu; 2004.

7. Sanvito WL. Propedêutica neurológica básica. $2^{\underline{a}}$ ed. São Paulo: Atheneu; 2010.

8. Rothi LJG, Ochipa C, Heilman KM. A cognitive neuropsychological model of limb praxis. Cogn Neuropsychol. 1991;8(6):443 58. http:// dx.doi.org/10.1080/02643299108253382

9. Rodrigues JC, Pawlowski J, Zibetti MR, Fonseca RP, Parente MAMP. Avaliação de apraxias em pacientes com lesão cerebrovascular no hemisfério esquerdo. Psicol Teor Prát. 2011;13(2):209-20.

10. Rubinstein W, Politis D. Revisón de los patrones de alteración práxicos encontrados en diferentes tipos de demência, sobre la base de un modelo cognitivo. Rev Esp Neuropsicol. 2005;2(4):167-85.

11. Zadikoff C, Lang AE. Apraxia in movement disorders. Brain. 2005;128(Pt 7):1480-97. http://dx.doi.org/10.1093/brain/awh560

12. Buchman AS, Bennett DA. Loss of motor function in preclinical Alzheimer's disease. Expert Rev Neurother. 2011;11(5):665-76. http://dx.doi.org/10.1586/ern.11.57

13. Sá F, Pinto P, Cunha C, Lemos R, Letra L, Simões M, Santana I. Differences between Early and Late-Onset Alzheimer's Disease in
Neuropsychological Tests. Front Neurol. 2012;3:81. http://dx.doi. org/10.3389/fneur.2012.00081

14. American Psychiatric Association. Diagnostic and Statistical Manual of Mental Disorders - DSM-5. $5^{\text {th }}$ ed. Washington, DC: APA; 2014.

15. Ozkan S, Adapinar DO, Elmaci NT, Arslantas D. Apraxia for differentiation Alzheimer's disease from subcortical vascular dementia and mild cognitive impairment. Neuropsychiatr Dis Treat. 2013;9:947-51. http://dx.doi.org/10.2147/NDT.S47879

16. Helmes E. Cognitive screening of older adults: the utility of pentagon drawing. Int Psychogeriatr. 2013;25(3):413-9. http://dx.doi. org/10.1017/S1041610212001998

17. Lee SY, Oh SW. Visuoperceptual and constructive ability disturbances of patients with traumatic brain injury in Hutt Adaptation of the Bender Gestalt Test. Korean J Clin Psychol. 1998;17(1):311-7.

18. Johnen A, Frommeyer J, Modes F, Wiendl H, Duning T, Lohmann H. Dementia Apraxia Test (DATE): A Brief Tool to Differentiate Behavioral Variant Frontotemporal Dementia from Alzheimer's Dementia Based on Apraxia Profiles. J Alzheimers Dis. 2015;49(3):593-605. http://dx.doi.org/10.3233/JAD-150447

19. Roth M, Tym E, Mountjoy CQ, Huppert FA, Hendrie H, Verma S, Goddard R. CAMDEX. A standardised instrument for the diagnosis of mental disorder in the elderly with special reference to the early detection of dementia. Br J Psychiatry. 1986;149:698-709. http:// dx.doi.org/10.1192/bjp.149.6.698

20. Folstein MF, Folstein SE, McHugh PR. Mini-Mental State: a practical method for grading the cognitive state of patients for the clinician. J Psychiatr Res. 1975;12(3):189-98. http://dx.doi.org/10.1016/00223956(75)90026-6

21. Yesavage JA, Brink TL, Rose TL, Lum O, Huang V, Adey M, Leirer VO. Development and validation of a geriatric depression screening scale: a preliminary report. J Psychiatr Res. 1982-1983;17(1):37-49. http://dx.doi.org/10.1016/0022-3956(82)90033-4

22. Pfeffer RI, Kurosaki TT, Harrah CH Jr, Chance JM, Filos S. Measurement of functional activities in older adults in the community. J Gerontol. 1982;37(3):323-9. http://dx.doi.org/10.1093/geronj/37.3.323

23. Brucki SMD, Nitrini R, Caramelli P, Bertolucci PHF, Okamoto I. Sugestões para o Uso do Mini-Exame do Estado Mental no Brasil. Arq Neuropsiquiatr. 2003;61(3-B):777-81. http://dx.doi.org/10.1590/ S0004-282X2003000500014

24. McKhann GM, Knopman DS, Chertkow H, Hyman BT, Jack CR Jr, Kawas CH, Klunk WE, Koroshetz WJ, Manly JJ, Mayeux R, Mohs RC, Morris JC, Rossor MN, Scheltens P, Carrillo MC, Thies B, Weintraub $\mathrm{S}$, Phelps $\mathrm{CH}$. The diagnosis of dementia due to Alzheimer's disease: recommendations from the National Institute on Aging-Alzheimer's Association workgroups on diagnostic guidelines for Alzheimer's disease. Alzheimers Dement. 2011;7(3):263-9. http://dx.doi. org/10.1016/j.jalz.2011.03.005

25. Yesavage JA, Brooks JO 3rd, Taylor J, Tinklenberg J. Development of aphasia, apraxia and agnosia and decline in Alzheimer's disease. Am J Psychiatry. 1993;150(5):742-7. http://dx.doi.org/10.1176/ ajp.150.5.742

26. Ward M, Cecato JF, Aprahamian I, Martinelli JE. Assessment for apraxia in Mild Cognitive Impairment and Alzheimer's disease. Dement Neuropsychol. 2015;9(1):71-5. http://dx.doi.org/10.1590/ S1980-57642015DN91000011 